\title{
Analysis of Food Packaging Layered Polymers by SEM/EDS and Raman Spectroscopy
}

\author{
John Konopka ${ }^{1}$, Mark Wall ${ }^{1}$
}

${ }^{1}$ Thermo Fisher Scientific, 5225 Verona Rd., Madison WI 53711

Food packaging can consist of many layers of materials each engineered for different purposes. The layers may be very thin, in some cases much less than one micron. Principally composed of polymers layers may be composed of inorganics and inorganic particles may be embedded by design or as artifacts. Analysis for quality assurance or failure analysis is difficult due to the complex nature of the sample.

Raman can easily identify the polymers used in these products. Raman mapping has a resolution of one micron or better. SEM imaging easily achieves a resolution of ten nanometers and can distinguish different polymers by their appearance in electron imaging. EDS achieves a spatial resolution of a few tens of nanometers. It can identify all elements present here except hydrogen.

Together these techniques provide complementary information. In addition imaging and elemental mapping with SEM/EDS may be faster than Raman mapping.

For this work a JEOL JSM-7610F FESEM equipped with a Thermo Scientific NS7 EDS analyzer and $60 \mathrm{~mm} 2$ area Ultradry silicon drift detector were used for the SEM/EDS data. A Thermo Scientific DXRxi Raman spectrometer with optical microscope was used for the Raman spectroscopy.

The SEM conditions were $10 \mathrm{kV}$ acceleration voltage and about $3 \mathrm{nA}$ beam current.

The sample used here is a cross section of a commercially available potato chips bag. The sample was sectioned by a fresh razor blade. For SEM/EDS the sample was carbon coated.

Figure 1 compares the results from both SEM/EDS and Raman. In this view the interior of the package is towards the top of the image. The right image shows a Backscatter Electron (BSE) image of the cross section overlaid by elemental maps for $\mathrm{Al}, \mathrm{Ti}, \mathrm{Si}$ and $\mathrm{O}$. Twelve layers were identified by inspection ranging from about 25 microns to approximately $175 \mathrm{~nm}$ thick. Some are not visible at this low magnification view. An Al layer about $200 \mathrm{~nm}$ thick was seen. A $175 \mathrm{~nm}$ thick layer containing S and $\mathrm{Cl}$ was found (not shown in the figure). Several layers contained Ti rich particles. Some particles of silicon oxide, possibly artifacts, were seen. The live time for this map was 931 seconds.

The image at left in Figure 1 shows the results of the Raman mapping analysis. The identified polymers layers and their thicknesses are labeled. In general the two techniques agree well on the overall composition of the sample. The Raman analysis identifies the polymers making up each of the layers which was not possible by SEM/EDS. It also found the inorganic rutile particles embedded in some of the layers. It did not find any of the sub $200 \mathrm{~nm}$ layers seen in the SEM. 
For future work ion polishing will be used to remove surface topography that may be obscuring some fine details in the SEM/EDS results.

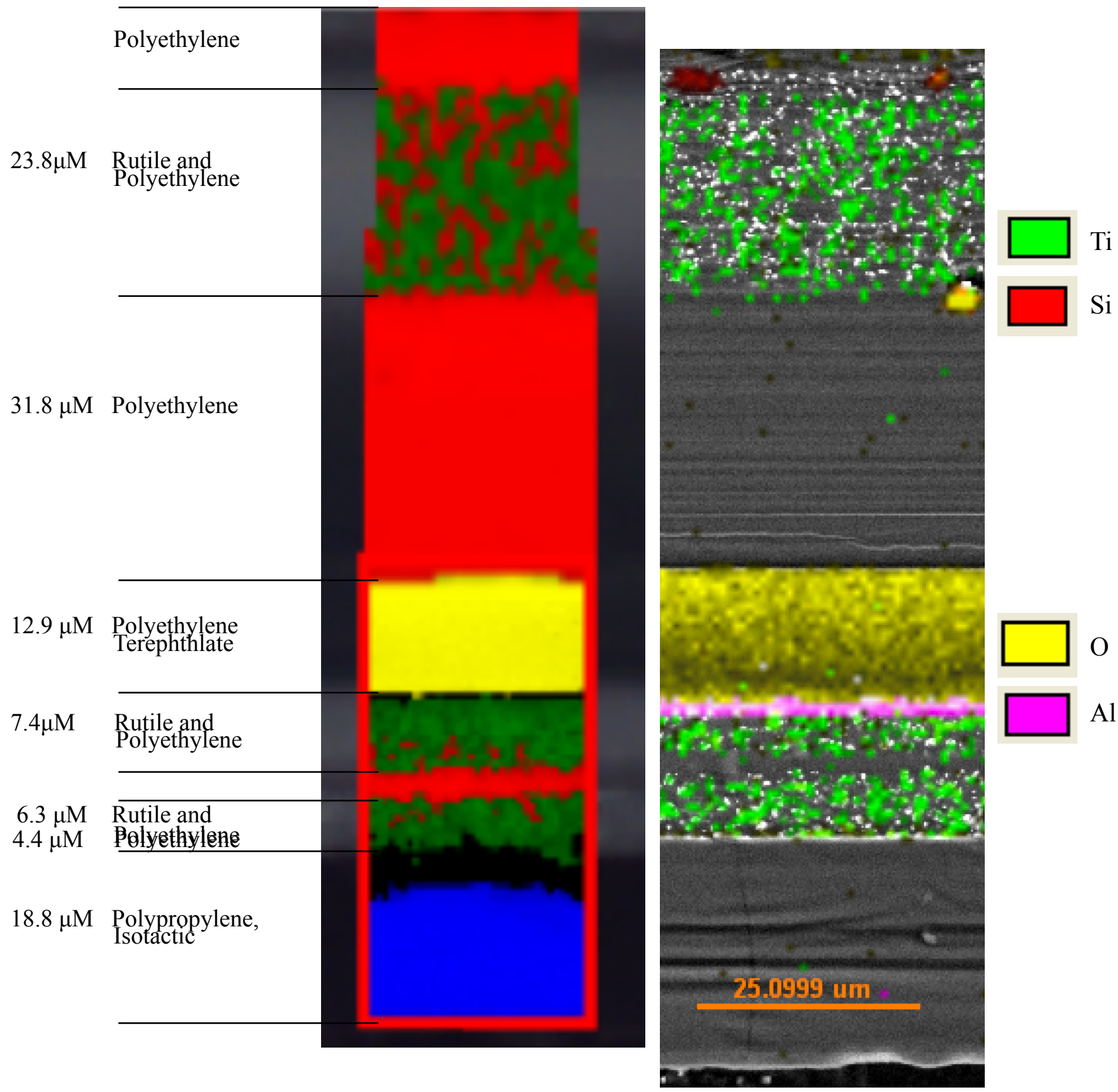

Figure 1. Two views of the same food package collected at different areas. The left image is produced by Raman analysis using a scanned laser through an optical microscope. The right side image is a BSE image from an SEM overlaid with elemental maps for Ti, Si, O and Al. The micron marker is only precise to about one micron. These are cross sectional images with the interior of the package at the top of the image. 